\title{
An Analytical and numerical investigation of escape rate for a noise driven bath
}

\author{
Jyotipratim Ray Chaudhuri*, Suman Kumar Banik, Bidhan Chandra Bag and \\ Deb Shankar Ray ${ }^{\dagger}$ \\ Indian Association for the Cultivation of Science, Jadavpur, Calcutta 700 032, India.
}

(October 23, 2018)

\begin{abstract}
We consider a system-reservoir model where the reservoir is modulated by an external noise. Both the internal noise of the reservoir and the external noise are stationary, Gaussian and are characterized by arbitrary decaying correlation functions. Based on a relation between the dissipation of the system and the response function of the reservoir driven by external noise we numerically examine the model using a full bistable potential to show that one can recover the turn-over features of the usual Kramers' dynamics when the external noise modulates the reservoir rather than the system directly. We derive the generalized Kramers' rate for this nonequilibrium open system. The theoretical results are verified by numerical simulation.
\end{abstract}

PACS number(s) : 05.40.-a, 02.50.Ey

* present address : Department of Physics, Katoa College, Katoa, Burdwan, West Bengal, India.

†e-mail : pcdsr@mahendra.iacs.res.in 


\section{INTRODUCTION}

More than half a century ago Kramers proposed a diffusion model for chemical reactions in terms of the theory of Brownian motion in phase space [1]. Since then the model and several of its variants have been ubiquitous in many areas of physics, chemistry and biology for understanding the nature of activated processes in classical [2 [7], quantum and semiclassical [8 11] systems, in general. These have become the subject of several reviews [12 14] and monograph [15] in the recent past.

In the majority of these treatments one is essentially concerned with an equilibrium thermal bath at a finite temperature which stimulates the reaction co-ordinate to cross the activation energy barrier. The inherent noise of the medium is of internal origin. This implies that the dissipative force which the system experiences in course of its motion in the medium and the stochastic force acting on the system as a result of random impact from the constituents of the medium arise from a common mechanism. From a microscopic point of view the system-reservoir Hamiltonian description [16 19] developed over the decades suggests that the coupling of the system and the reservoir co-ordinates determines both the noise and the dissipative terms in the Langevin equation describing the motion of the system. It is therefore not difficult to anticipate that these two entities get related through a fluctuation-dissipation relation [20] ( these systems are sometimes classified as thermodynamically closed system in contrast to the systems driven by external noise in nonequilibrium statistical mechanics 21] ). However, when the reservoir is modulated by an external noise it is likely that this relation gets affected in a significant way. Since the modulation of the reservoir crucially depend on its response function, one can further envisage a connection between the dissipation of the system and the response function of the reservoir due to the external noise from a microscopic point of view.

In the present paper we explore this connection in the context of activated rate processes when the reservoir is modulated by an external noise. Specifically our object here is twofold: (i) to explore the role of reservoir response as a function of external noise on the system 
dynamics in contrast to direct driving of the system by external noise, (ii) to calculate the generalized Kramers' rate for the steady state of this nonequilibrium open system taking full care of thermodynamic consistency condition. Both the internal and the external noises are Gaussian, stationary and are characterized by arbitrary decaying correlation functions. While the internal noise of the reservoir is thermal, the external noise may be of thermal or non-thermal type. We consider the stochastic motion to be spatial diffusion limited and calculate the rate of escape in the intermediate to strong damping regime. It is worth to mention that the externally generated nonequilibrium fluctuations can bias the Brownian motion of a particle in an anisotropic medium and may be used for designing molecular motors and pumps [22]. We further mention that nonequilibrium, non-thermal systems have also been investigated phenomenologically by a number of workers in several other contexts, e.g., for examining the role of color noise in stationary probabilities [23], properties of nonlinear systems [24], nature of crossover [25], rate of diffusion limited coagulation processes [26], effect of monochromatic noise [27], etc. While these treatments concern direct driving of the system by an external noise the present consideration is based on modulation of the bath. A number of different situations depicting the modulation of the bath by an external noise may be physically relevant. As, for example, we consider a simple unimolecular conversion (say, an isomerization reaction) from $A \longrightarrow B$. The reaction is carried out in a photochemically active solvent under the influence of external fluctuating light intensity. Since the fluctuations in the light intensity result in the fluctuations in the polarization of the solvent molecules, the effective reaction field around the reactant system gets modified. Provided the required stationarity of this nonequilibrium open system is maintained (which is not difficult in view of the experiments performed in the studies of external noise-induced transitions in photochemical systems [28]) the dynamics of barrier crossing becomes amenable to the present theoretical analysis that follows.

The remaining part of this paper is organized as follows: In Sec. II we discuss a systemreservoir model where the later is modulated by an external noise and establish an interesting connection between the dissipation of the system and the response function of the reservoir 
due to external noise. The stochastic motion in a linearized potential field is described in terms of a Fokker-Planck equation in Sec. III. Based on the traditional flux over population

method [29] we derive in Sec. IV the generalized expression for the Kramers' rate of escape from a metastable well. In Sec. V we numerically analyze the model and the bath modulated dynamics for the full potential and verify the theoretical rate with numerical simulation. The paper is concluded in Sec. VI.

\section{THE SYSTEM-RESERVOIR MODEL : THE RESERVOIR MODULATED BY AN EXTERNAL NOISE}

We consider a classical particle of mass $M$ is linearly coupled to a heat bath of $N$ harmonic oscillators driven by an external noise. The total Hamiltonian is given by [18]

$$
H=\frac{p^{2}}{2 M}+V(x)+\frac{1}{2} \sum_{i=1}^{N}\left\{\frac{p_{i}^{2}}{m_{i}}+m_{i} \omega_{i}^{2}\left(q_{i}-g_{i} x\right)^{2}\right\}+H_{\text {int }}
$$

In Eq.(11), $x$ and $p$ are the co-ordinate and momentum of the system particle; $\left(q_{i}, p_{i}\right)$ are the variables associated with the $i$-th oscillator and $\omega_{i}$ and $m_{i}$ are the corresponding frequency and mass, respectively. $g_{i} x$ measures the interaction between the particle and the bath. $V(x)$ is the potential energy of the particle. $H_{\text {int }}$ is assumed to be of the form

$$
H_{\text {int }}=\frac{1}{2} \sum_{i=1}^{N} \kappa_{i} q_{i} \epsilon(t)
$$

The coupling function $\kappa_{i}$ measures the strength of interaction and $\epsilon(t)$ is the external noise which we assume to be stationary and Gaussian with zero mean, i.e., $\langle\epsilon(t)\rangle_{e}=0$ and is characterized by an arbitrary correlation function as follows;

$$
\left\langle\epsilon(t) \epsilon\left(t^{\prime}\right)\right\rangle_{e}=2 D \Psi\left(t-t^{\prime}\right)
$$

Here $\langle\ldots\rangle_{e}$ implies the averaging over the external noise.

We then eliminate the bath degrees of freedom in the usual way [16, 18, 19, 30 to obtain the following generalized Langevin equation 


$$
\begin{aligned}
& \dot{x}=v \\
& \dot{v}=-\frac{d V}{d x}-\int_{0}^{t} d t^{\prime} \gamma\left(t-t^{\prime}\right) v\left(t^{\prime}\right)+f(t)+\pi(t)
\end{aligned}
$$

[ while constructing Eq.(3) we have set $M$ and $m_{i}$ equal to unity ] where

$$
\gamma(t)=\sum_{i=1}^{N} g_{i}^{2} \omega_{i}^{2} \cos \omega_{i} t
$$

$f(t)$ is the internal forcing function generated through the coupling between the system and the heat bath and is given by

$$
f(t)=\sum_{i=1}^{N} g_{i}\left\{\left[q_{i}(0)-g_{i} x(0)\right] \omega_{i}^{2} \cos \omega_{i} t+v_{i}(0) \omega_{i} \sin \omega_{i} t\right\}
$$

In Eq.(3), $\pi(t)$ is a fluctuating force term due to the external noise $\epsilon(t)$ and is given by

$$
\pi(t)=-\int_{0}^{t} \varphi\left(t-t^{\prime}\right) \epsilon\left(t^{\prime}\right) d t^{\prime}
$$

where

$$
\varphi(t)=\sum_{i=1}^{N} g_{i} \kappa_{i} \omega_{i} \sin \omega_{i} t
$$

The form of Eq.(3) therefore suggests that the system is driven by two forcing functions $f(t)$ and $\pi(t) . f(t)$ depends on the initial conditions of the bath oscillators for a fixed choice of the initial condition of the system degrees of freedom. To define the statistical properties of $f(t)$, we assume that the initial distribution is one in which the bath is equilibrated at $t=0$ in the presence of the system but in the absence of the external noise agency such that $\langle f(t)\rangle=0$ and $\left\langle f(t) f\left(t^{\prime}\right)\right\rangle=k_{B} T \gamma\left(t-t^{\prime}\right)$.

Now, at $t=0_{+}$, the external noise agency is switched on and the bath is modulated by $\epsilon(t)$. The system is governed by Eq.(3), where, apart from the internal noise $f(t)$, another fluctuating force $\pi(t)$ appears, that depends on the external noise $\epsilon(t)$. Therefore, one can define an effective noise $\xi(t)(=f(t)+\pi(t))$ whose correlation is given by

$$
\left\langle\left\langle\xi(t) \xi\left(t^{\prime}\right)\right\rangle\right\rangle=k_{B} T \gamma\left(t-t^{\prime}\right)+2 D \int_{0}^{t} d t^{\prime} \int_{0}^{t^{\prime}} d t^{\prime \prime} \varphi\left(t-t^{\prime}\right) \varphi\left(t^{\prime}-t^{\prime \prime}\right) \Psi\left(t^{\prime}-t^{\prime \prime}\right)
$$


along with $\langle\langle\xi(t)\rangle\rangle=0$, where $\langle\langle\ldots\rangle\rangle$ means we have taken two averages independently. It should be emphasized that the above relation (8) is not a fluctuation-dissipation relation due to the appearance of the external noise intensity. Rather it serves as a thermodynamic consistency condition.

Let us now digress a little bit about $\pi(t)$. The statistical properties of $\pi(t)$ are determined by the normal mode density of the bath frequencies, the coupling of the system with the bath, the coupling of the bath with the external noise and the external noise itself. Eq.(6) is reminiscent of the familiar linear relation between the polarization and the external field where $\pi$ and $\epsilon$ play the role of the former and the later, respectively. $\varphi(t)$ can then be interpreted as a response function of the reservoir due to external noise $\epsilon(t)$. The very structure of $\pi(t)$ suggests that this forcing function although originating from an external force is different from a direct driving force acting on the system. The distinction lies at the very nature of the bath characteristics (rather than system characteristics) as reflected in the relation (6) and (7).

With the coupling coefficients $g(\omega)=g_{0} / \sqrt{\tau_{c}} \omega$ and $\kappa(\omega)=\sqrt{\tau_{c}} \omega \kappa_{0}$, in the continuum limit [30] $\gamma(t)$ and $\varphi(t)$ reduce to the following forms

$$
\gamma(t)=\frac{g_{0}^{2}}{\tau_{c}} \int d \omega \mathcal{D}(\omega) \cos \omega t
$$

and

$$
\varphi(t)=g_{0} \kappa_{0} \int d \omega \mathcal{D}(\omega) \omega \sin \omega t
$$

where $g_{0}$ and $\kappa_{0}$ are constants and $\tau_{c}^{-1}$ is the cutoff frequency of the oscillator. $\mathcal{D}(\omega)$ is the density of modes of the heat bath.

From the above two relations, we obtain

$$
\frac{d \gamma}{d t}=-\frac{g_{0}}{\kappa_{0}} \frac{1}{\tau_{c}} \varphi(t)
$$

Eq.(11) is an important content of the present model. This expresses how the dissipative kernel $\gamma(t)$ depends on the response function $\varphi(t)$ of the medium due to external noise $\epsilon(t)$ 
[ see Eq.(6) ]. Such a relation for the open system can be anticipated in view of the fact that both the dissipation and the response function crucially depend on the properties of the reservoir especially on its density of modes and its coupling to the system and the external noise source. In what follows we shall be concerned with the consequences of this relation in terms of the Langevin description in the next section ( Eq.(12) ) and numerical analysis of the full model potential in Sec. V.

\section{THE FOKKER-PLANCK DESCRIPTION OF THE LINEARIZED MOTION : ASYMPTOTIC ANALYSIS OF THE FOKKER-PLANCK COEFFICIENTS}

We now consider the system to be a harmonically bound particle of unit mass and of frequency $\omega_{0}$. Then because of Eq.(河) the Langevin equation (3) becomes

$$
\begin{aligned}
& \dot{x}=v \\
& \dot{v}=-\omega_{0}^{2} x-\int_{0}^{t} d t^{\prime} \gamma\left(t-t^{\prime}\right) v\left(t^{\prime}\right)+f(t)-\int_{0}^{t} d t^{\prime} \varphi\left(t-t^{\prime}\right) \epsilon\left(t^{\prime}\right)
\end{aligned}
$$

The Laplace transform of Eq. (12) allows us to write a formal solution for the displacement of the form

$$
\begin{aligned}
x(t)= & \langle\langle x(t)\rangle\rangle+\int_{0}^{t} d t^{\prime} h\left(t-t^{\prime}\right) f\left(t^{\prime}\right)-\frac{\kappa_{0}}{g_{0}} \tau_{c} \omega_{0}^{2} \int_{0}^{t} d t^{\prime} h\left(t-t^{\prime}\right) \epsilon\left(t^{\prime}\right) \\
& -\frac{\kappa_{0}}{g_{0}} \tau_{c} \int_{0}^{t} d t^{\prime} h_{2}\left(t-t^{\prime}\right) \epsilon\left(t^{\prime}\right),
\end{aligned}
$$

where we have made use of the relation (11) explicitly.

Here

$$
\langle\langle x(t)\rangle\rangle=\chi_{x}(t) x(0)+h(t) v(0)
$$

with $x(0)$ and $v(0)$ being the initial position and initial velocity of the oscillator, respectively, which are nonrandom and

$$
\chi_{x}(t)=\left[1-\omega_{0}^{2} \int_{0}^{t} h(\tau) d \tau\right]
$$


The kernel $h(t)$ is the Laplace inversion of

$$
\tilde{h}(s)=\frac{1}{s^{2}+\tilde{\gamma}(s) s+\omega_{0}^{2}}
$$

where, $\tilde{\gamma}(s)=\int_{0}^{\infty} e^{-s t} \gamma(t) d t$, is the Laplace transform of the friction kernel $\gamma(t)$, and

$$
h_{2}(t)=\frac{d^{2} h(t)}{d t^{2}} .
$$

The time derivative of Eq.(13) yields

$$
\begin{aligned}
v(t)= & \langle\langle v(t)\rangle\rangle+\int_{0}^{t} d t^{\prime} h_{1}\left(t-t^{\prime}\right) f\left(t^{\prime}\right)-\frac{\kappa_{0}}{g_{0}} \tau_{c} \omega_{0}^{2} \int_{0}^{t} d t^{\prime} h_{1}\left(t-t^{\prime}\right) \epsilon\left(t^{\prime}\right) \\
& -\frac{\kappa_{0}}{g_{0}} \tau_{c} \int_{0}^{t} d t^{\prime} h_{3}\left(t-t^{\prime}\right) \epsilon\left(t^{\prime}\right)
\end{aligned}
$$

where

$$
\begin{gathered}
\langle\langle v(t)\rangle\rangle=-\omega_{0}^{2} h(t)+v(0) h_{1}(t), \\
h_{1}(t)=\frac{d h(t)}{d t} \text { and } h_{3}(t)=\frac{d^{3} h(t)}{d t^{3}} .
\end{gathered}
$$

Next we calculate the variances. From the formal solution of $x(t)$ and $v(t)$, the explicit expressions for the variances are obtained which are given below;

$$
\begin{aligned}
\sigma_{x x}^{2}(t)= & \left\langle\left\langle[x(t)-\langle\langle x(t)\rangle\rangle]^{2}\right\rangle\right\rangle \\
= & 2 \int_{0}^{t} d t_{1} h\left(t_{1}\right) \int_{0}^{t_{1}} d t_{2} h\left(t_{2}\right)\left\langle f\left(t_{1}\right) f\left(t_{2}\right)\right\rangle \\
& +2\left(\frac{\kappa_{0}}{g_{0}} \tau_{c} \omega_{0}^{2}\right)^{2} \int_{0}^{t} d t_{1} h\left(t_{1}\right) \int_{0}^{t_{1}} d t_{2} h\left(t_{2}\right)\left\langle\epsilon\left(t_{1}\right) \epsilon\left(t_{2}\right)\right\rangle_{e} \\
& +2\left(\frac{\kappa_{0}}{g_{0}} \tau_{c}\right)^{2} \int_{0}^{t} d t_{1} h_{2}\left(t_{1}\right) \int_{0}^{t_{1}} d t_{2} h_{2}\left(t_{2}\right)\left\langle\epsilon\left(t_{1}\right) \epsilon\left(t_{2}\right)\right\rangle_{e} \\
& +2\left(\frac{\kappa_{0}}{g_{0}} \tau_{c}\right)^{2} \omega_{0}^{2} \int_{0}^{t} d t_{1} h\left(t_{1}\right) \int_{0}^{t_{1}} d t_{2} h_{2}\left(t_{2}\right)\left\langle\epsilon\left(t_{1}\right) \epsilon\left(t_{2}\right)\right\rangle_{e}, \\
\sigma_{v v}^{2}(t)= & \left\langle\left\langle[v(t)-\langle\langle v(t)\rangle\rangle]^{2}\right\rangle\right\rangle \\
= & 2 \int_{0}^{t} d t_{1} h_{1}\left(t_{1}\right) \int_{0}^{t_{1}} d t_{2} h_{1}\left(t_{2}\right)\left\langle f\left(t_{1}\right) f\left(t_{2}\right)\right\rangle
\end{aligned}
$$




$$
\begin{aligned}
& +2\left(\frac{\kappa_{0}}{g_{0}} \tau_{c} \omega_{0}^{2}\right)^{2} \int_{0}^{t} d t_{1} h_{1}\left(t_{1}\right) \int_{0}^{t_{1}} d t_{2} h_{1}\left(t_{2}\right)\left\langle\epsilon\left(t_{1}\right) \epsilon\left(t_{2}\right)\right\rangle_{e} \\
& +2\left(\frac{\kappa_{0}}{g_{0}} \tau_{c}\right)^{2} \int_{0}^{t} d t_{1} h_{3}\left(t_{1}\right) \int_{0}^{t_{1}} d t_{2} h_{3}\left(t_{2}\right)\left\langle\epsilon\left(t_{1}\right) \epsilon\left(t_{2}\right)\right\rangle_{e} \\
& +2\left(\frac{\kappa_{0}}{g_{0}} \tau_{c}\right)^{2} \omega_{0}^{2} \int_{0}^{t} d t_{1} h_{1}\left(t_{1}\right) \int_{0}^{t_{1}} d t_{2} h_{3}\left(t_{2}\right)\left\langle\epsilon\left(t_{1}\right) \epsilon\left(t_{2}\right)\right\rangle_{e}
\end{aligned}
$$

and

$$
\begin{aligned}
\sigma_{x v}^{2}(t) & =\langle\langle[x(t)-\langle\langle x(t)\rangle\rangle][v(t)-\langle\langle v(t)\rangle\rangle]\rangle\rangle \\
& =\frac{1}{2} \dot{\sigma}_{x x}^{2}(t)
\end{aligned}
$$

where we have assumed that the noises $f(t)$ and $\epsilon(t)$ are symmetric with respect to the time argument and have made use the fact that $f(t)$ and $\epsilon(t)$ are uncorrelated.

Due to the Gaussian property of the noises $f(t)$ and $\epsilon(t)$ and the linearity of the Langevin equation (12), we see that the joint probability density $p(x, v, t)$ of the oscillator must be Gaussian. The joint characteristic function associated with the density is

$$
\tilde{p}(\mu, \rho, t)=\exp \left\{i\langle\langle x(t)\rangle\rangle \mu+i\langle\langle v(t)\rangle\rangle \rho-\frac{1}{2}\left[\sigma_{x x}^{2}(t) \mu^{2}+2 \sigma_{x v}^{2}(t) \rho \mu+\sigma_{v v}^{2}(t) \rho^{2}\right]\right\} .
$$

Using the method of characteristic function [31,32 and the above expression (24) we find the general Fokker-Planck equation associated with the probability density function $p(x, v, t)$ for the process (12);

$$
\frac{\partial p}{\partial t}=-v \frac{\partial p}{\partial x}+\bar{\omega}_{0}^{2}(t) x \frac{\partial p}{\partial v}+\bar{\gamma}(t) \frac{\partial}{\partial v}(v p)+\phi(t) \frac{\partial^{2} p}{\partial v^{2}}+\psi(t) \frac{\partial^{2} p}{\partial v \partial x}
$$

where

$$
\begin{aligned}
& \bar{\gamma}(t)=-\frac{d}{d t} \ln \Upsilon(t), \quad \bar{\omega}_{0}^{2}(t)=\frac{-h(t) h_{1}(t)+h_{1}^{2}(t)}{\Upsilon(t)} \text { and } \\
& \Upsilon(t)=\frac{h_{1}(t)}{\omega_{0}^{2}}\left[1-\omega_{0}^{2} \int_{0}^{\tau} d \tau h(\tau)\right]+h^{2}(t)
\end{aligned}
$$

The functions $\phi(t)$ and $\psi(t)$ are defined by

$$
\phi(t)=\bar{\omega}_{0}^{2}(t) \sigma_{x v}^{2}+\bar{\gamma} \sigma_{v v}^{2}+\frac{1}{2} \dot{\sigma}_{x v}^{2} \text { and } \psi(t)=\dot{\sigma}_{x v}^{2}+\bar{\gamma}(t) \sigma_{x v}^{2}+\bar{\omega}_{0}^{2} \sigma_{x x}^{2}-\sigma_{v v}^{2}
$$


where the covariances are to be calculated for a particular given noise process.

For the internal noise processes it had been shown earlier that for several models the various time dependent parameters $\bar{\omega}_{0}^{2}(t), \bar{\gamma}(t)$, etc. do exist asymptotically as $t \rightarrow \infty$. The above consideration shows that $h(t), h_{1}(t)$, etc. do not depend on the nature of the noise but depend only on the relaxation $\bar{\gamma}(t)$.

We now discuss the asymptotic properties of $\phi(t)$ and $\psi(t)$, which in turn are dependent on the variances $\sigma_{x x}^{2}(t)$ and $\sigma_{v v}^{2}(t)$, as $t \rightarrow \infty$ since they play a significant role in our further analysis that follows.

From Eqs. (21) and (22), we may write

$$
\sigma_{x x}^{2}(t)=\sigma_{x x}^{2(i)}(t)+\sigma_{x x}^{2(e)}(t) \text { and } \sigma_{v v}^{2}(t)=\sigma_{v v}^{2(i)}(t)+\sigma_{v v}^{2(e)}(t)
$$

where ' $i$ ' denotes the part corresponding to internal noise $f(t)$ and ' $e$ ' corresponds to the external noise $\epsilon(t)$. Since the average velocity of the oscillator is zero as $t \rightarrow \infty$ we see from Eq.(19) that $h(t)$ and $h_{1}(t)$ must be zero as $t \rightarrow \infty$. Also from Eq.(14) we observe that the function $\chi_{x}(t)$ must decay to zero for long times. Hence, from Eq.(15) we see that the stationary value of the integral of $h(t)$ is $1 / \omega_{0}^{2}$, i.e.,

$$
\int_{0}^{\infty} h(t) d t=\frac{1}{\omega_{0}^{2}}
$$

Now, $\sigma_{x x}^{2(i)}(t)$ and $\sigma_{v v}^{2(i)}(t)$ of Eqs. 21) and (22) can be written in the form

$$
\begin{aligned}
\sigma_{x x}^{2(i)}(t) & =2 \int_{0}^{t} d t_{1} h\left(t_{1}\right) \int_{0}^{t_{1}} d t_{2} h\left(t_{2}\right)\left\langle f\left(t_{1}\right) f\left(t_{2}\right)\right\rangle \\
& =k_{B} T\left[2 \int_{0}^{t} d \tau h(\tau)-h^{2}(t)-\omega_{0}^{2}\left\{\int_{0}^{t} d \tau h(\tau)\right\}^{2}\right]
\end{aligned}
$$

and

$$
\begin{aligned}
\sigma_{v v}^{2(i)}(t) & =2 \int_{0}^{t} d t_{1} h_{1}\left(t_{1}\right) \int_{0}^{t_{1}} d t_{2} h_{1}\left(t_{2}\right)\left\langle f\left(t_{1}\right) f\left(t_{2}\right)\right\rangle \\
& =k_{B} T\left[1-h_{1}^{2}(t)-\omega_{0}^{2} h^{2}(t)\right] .
\end{aligned}
$$

From the above two expressions [ Eqs.(28) and (29) ] we see that 


$$
\sigma_{x x}^{2(i)}(\infty)=\frac{k_{B} T}{\omega_{0}^{2}} \quad \text { and } \quad \sigma_{v v}^{2(i)}(\infty)=k_{B} T .
$$

It is important to note that these stationary values are not related to the intensity and correlation time of the internal noise.

We next consider the parts, $\sigma_{x x}^{2(e)}(t)$ and $\sigma_{v v}^{2(e)}(t)$, due to the presence of the external noise. The Laplace transform of Eq.(13) yields the expression

$$
\tilde{x}(s)-\langle\langle\tilde{x}(s)\rangle\rangle=\tilde{h}(s) \tilde{f}(s)-\frac{\kappa_{0}}{g_{0}} \tau_{c} \omega_{0}^{2} \tilde{h}(s) \tilde{\epsilon}(s)-\frac{\kappa_{0}}{g_{0}} \tau_{c} s^{2} \tilde{h}(s) \tilde{\epsilon}(s)
$$

where

$$
\begin{aligned}
\langle\langle\tilde{x}(s)\rangle\rangle & =\left\{\frac{1}{s}-\frac{\omega_{0}^{2}}{s\left[s^{2}+s \tilde{\gamma}(s)+\omega_{0}^{2}\right]}\right\} x(0)+\frac{1}{s^{2}+s \tilde{\gamma}(s)+\omega_{0}^{2}} v(0) \\
& =\left\{\frac{1}{s}-\omega_{0}^{2} \frac{\tilde{h}(s)}{s}\right\} x(0)+\tilde{h}(s) v(0) .
\end{aligned}
$$

From the above equation (31) we can calculate the variance $\sigma_{x x}^{2}$ in the Laplace-transformed space which can be identified as the Laplace transform of Eq.(21). Thus, for the part $\sigma_{x x}^{2(e)}(t)$ we observe that, $\tilde{\sigma}_{x x}^{2(e)}(s)$ contains terms like $\left(\frac{\kappa_{0}}{g_{0}} \tau_{c} \omega_{0}^{2} \tilde{h}(s)\right)^{2}\left\langle\tilde{\epsilon}^{2}(s)\right\rangle_{e}$. Since, we have assumed the stationarity of the noise $\epsilon(t)$, we conclude that if $\tilde{C}(0)$ exists [ where $C\left(t-t^{\prime}\right)=\left\langle\epsilon(t) \epsilon\left(t^{\prime}\right)\right\rangle_{e}$ ], then the stationary value of $\sigma_{x x}^{2(e)}(t)$ exists and becomes a constant that depends on the correlation time and the strength of the noise. Similar argument is also valid for $\sigma_{v v}^{2(e)}(t)$.

Summarizing the above discussions we note that, (i) the internal noise-driven parts of $\sigma_{x x}^{2}(t)$ and $\sigma_{v v}^{2}(t)$, i.e., $\sigma_{x x}^{2(i)}$ and $\sigma_{v v}^{2(i)}$ approach the fixed values which are independent of the noise correlation and the intensity as $t \rightarrow \infty$,

(ii) the external noise driven parts of variances also approach the constant values at the stationary $(t \rightarrow \infty)$ limit which are dependent on the strength and the correlation time of the noise.

Hence we conclude, following the Ref.(33) and our preceding discussions that even in presence of an external noise the above terms do exist asymptotically and we write the steady state Fokker-Planck equation for the asymptotic values of the parameters as,

$$
-v \frac{\partial p}{\partial x}+\bar{\omega}_{0}^{2} x \frac{\partial p}{\partial v}+\bar{\gamma} \frac{\partial}{\partial v}(v p)+\phi(\infty) \frac{\partial^{2} p}{\partial v^{2}}+\psi(\infty) \frac{\partial^{2} p}{\partial v \partial x}=0
$$


where, $\bar{\omega}_{0}^{2}, \bar{\gamma}, \phi(\infty), \psi(\infty)$, etc. are to be calculated from the general definition (26) for the steady state. As an explicit example we consider the case of a $\delta$-correlated external noise and Ornstein-Uhlenbeck internal noise for which we provide the expressions for variances $\sigma_{x x}^{2}(t), \sigma_{v v}^{2}(t)$ and $\sigma_{x v}^{2}(t)$ and the relaxation function $h(t)$ given in the Appendix-A.

The general steady state solution of the above equation (33) is

$$
p_{s t}(x, v)=\frac{1}{Z} \exp \left[-\left\{\frac{v^{2}}{2 D_{0}}+\frac{\bar{\omega}_{0}^{2} x}{2\left(D_{0}+\psi(\infty)\right)}\right\}\right]
$$

where

$$
D_{0}=\frac{\phi(\infty)}{\bar{\gamma}}
$$

and $Z$ is the normalization constant. The solution (34) can be verified by direct substitution. The distribution (34) is not an equilibrium distribution. This stationary distribution for the

nonequilibrium open system plays the role of an equilibrium distribution of the closed system which may, however, be recovered in the absence of external noise term.

\section{KRAMERS' ESCAPE RATE}

We now turn to the problem of decay of a metastable state. In Kramers approach [1], the particle coordinate $x$ corresponds to the reaction coordinate and its values at the minima of the potential $V(x)$ denotes the reactant and product states.

Linearizing the motion around barrier top at $x=x_{b}$ the Langevin equation (3) can be written down as

$$
\begin{aligned}
& \dot{y}=v \\
& \dot{v}=\omega_{b}^{2} y-\int_{0}^{t} d t^{\prime} \gamma\left(t-t^{\prime}\right) v\left(t^{\prime}\right)+f(t)+\pi(t),
\end{aligned}
$$

where, $y=x-x_{b}$ and the barrier frequency $\omega_{b}^{2}$ is defined by

$$
V(y)=V_{b}-\frac{1}{2} \omega_{b}^{2} y^{2} ; \omega_{b}^{2}>0
$$

Correspondingly the motion of the particle is governed by the Fokker-Planck equation (25) 


$$
\frac{\partial p}{\partial t}=-v \frac{\partial p}{\partial y}-\bar{\omega}_{b}^{2}(t) y \frac{\partial p}{\partial v}+\bar{\gamma}_{b}(t) \frac{\partial}{\partial v}(v p)+\phi_{b}(t) \frac{\partial^{2} p}{\partial v^{2}}+\psi_{b}(t) \frac{\partial^{2} p}{\partial v \partial y},
$$

where, the suffix ' $b$ ' indicates that all the coefficients are to be calculated using the general definition (26) for the barrier top region.

It is apparent from Eqs.(33) and (38) that since the dynamics is non-Markovian and the system is thermodynamically open one has to deal with the renormalized frequencies $\bar{\omega}_{0}$ and $\bar{\omega}_{b}$ near the bottom or top of the well, respectively. We make the ansatz that the nonequilibrium, steady state probability $p_{b}$, generating a nonvanishing diffusion current $j$, across the barrier is given by

$$
p_{b}(x, v)=\exp \left[-\left\{\frac{v^{2}}{2 D_{b}}+\frac{\tilde{V}(x)}{D_{b}+\psi_{b}(\infty)}\right\}\right] \xi(x, v)
$$

where

$$
D_{b}=\frac{\phi_{b}(\infty)}{\bar{\gamma}_{b}}
$$

$\tilde{V}(x)$ is the renormalized linear potential as

$$
\begin{aligned}
& \tilde{V}(x)=V\left(x_{0}\right)+\frac{1}{2} \bar{\omega}_{0}^{2}\left(x-x_{0}\right)^{2}, \text { near the bottom } \\
& \tilde{V}(x)=V\left(x_{b}\right)-\frac{1}{2} \bar{\omega}_{b}^{2}\left(x-x_{b}\right)^{2}, \text { near the top }
\end{aligned}
$$

with $\bar{\omega}_{0}^{2}, \bar{\omega}_{b}^{2}>0$. The unknown function $\xi(x, v)$ obeys the natural boundary condition that for $x \rightarrow \infty, \xi(x, v)$ vanishes.

The ansatz of the form (39) denoting the steady state distribution is motivated by the local analysis near the bottom and the top of the barrier in the Kramers' sense [1]. For a stationary nonequilibrium system, on the other hand, the relative population of the two regions, in general, depends on the global properties of the potential leading to an additional factor in the rate expression. Although because of the Kramers' type ansatz [1] which is valid for the local analysis, such a consideration is outside the scope of the present treatment, we point out a distinctive feature in the ansatz (39) compared to Kramers' ansatz. While in the latter case one considers a complete factorization of the equilibrium part (Boltzmann) 
and the dynamical part, the ansatz (39) incorporates the additional dynamical contribution through dissipation and strength of the noise into the exponential part. This modification of Kramers' ansatz (by dynamics) is due to nonequilibrium nature of the system. Thus unlike Kramers', the exponential factors in (39) and in the stationary distribution (34) which serves as a boundary condition are markedly different. Before carrying out global analysis in the present section our aim here is to understand the modification of the rate due to modulation of the bath driven by an external noise, within the perview of Kramers' type ansatz. The internal consistency of the treatment, however, can be checked by recovering the Kramers' result when the external noise is switched off.

From equation (38), using (39) we obtain the equation for $\xi(y, v)$ in the steady state in the neighborhood of $x_{b}$, the equation

$$
-\left(1+\frac{\psi_{b}(\infty)}{D_{b}}\right) v \frac{\partial \xi}{\partial y}-\left[\frac{D_{b}}{D_{b}+\psi_{b}(\infty)} \bar{\omega}_{b}^{2} y+\bar{\gamma}_{b} v\right] \frac{\partial \xi}{\partial v}+\phi_{b}(\infty) \frac{\partial^{2} \xi}{\partial v^{2}}+\psi_{b}(\infty) \frac{\partial^{2} \xi}{\partial v \partial y}=0
$$

After making use of the appropriate transformations and boundary conditions for reduced distribution functions [7] we obtain the barrier crossing rate $k$ given by

$$
k=\frac{\bar{\omega}_{0}}{2 \pi} \frac{D_{b}}{\left\{D_{0}+\psi(\infty)\right\}^{1 / 2}}\left(\frac{\Lambda}{1+\Lambda D_{b}}\right)^{1 / 2} \exp \left[\frac{-E_{0}}{D_{b}+\psi_{b}(\infty)}\right]
$$

where

$$
\Lambda=\frac{\lambda}{\phi_{b}(\infty)+a \psi_{b}(\infty)}
$$

with

$$
a=\frac{D_{b}}{2\left(D_{b}+\psi_{b}(\infty)\right)}\left\{-\bar{\gamma}_{b}-\sqrt{\bar{\gamma}_{b}^{2}+4 \bar{\omega}_{b}^{2}}\right\} \text { and } \lambda=-\bar{\gamma}_{b}-a\left(1+\frac{\psi_{b}(\infty)}{D_{b}}\right)
$$

Here $E_{0}$ is the activation energy, $E_{0}=V\left(x_{b}\right)-V\left(x_{0}\right)$. Since the temperature due to internal thermal noise, the strength of the external noise and the damping constant are buried in the parameters $D_{0}, D_{b}, \psi_{0}, \psi_{0}$ and $\Lambda$ the generalized expression look somewhat cumbersome. We point out that the subscripts ' 0 ' and ' $b$ ' in $D$ and $\psi$ refer to the well or barrier top region, respectively. Eq.(43) is one of the key results of this paper. We note here that 
$\left(D_{b}+\psi_{b}(\infty)\right) / k_{B}$ in the exponential factor defines a new effective temperature characteristic of the steady state of the nonequilibrium open system and an effective transmission factor is contained in the prefactor controlling the barrier crossing dynamics. As expected both are the functions of the external noise strength $D$ and coupling of noise to the bath modes. The dependence of the rate on the parameters can be exposed explicitly once we consider the typical cases.

\section{NUMERICAL ANALYSIS OF THE MODEL AND THE BARRIER CROSSING RATE}

\section{A. The model : bath modulation vs. direct driving}

We first explore the distinctive aspects of the external noise-driven-reservoir-modulated dynamics of the system ( in contact with the external noise driven bath ) in contrast to direct driving of the system by the external noise. This will help us to elucidate the special role of the reservoir response function in controlling the rate. To this end we first return to our basic equation of the model, Eq.(3) where $\varphi\left(t-t^{\prime}\right)$ as given by Eq.(7) characterizes the response function. We solve this equation numerically using second order stochastic algorithm of Fox [34 for the full potential $V(x)=\frac{1}{4} x^{4}-\frac{1}{2} x^{2}$ and plot the results of computation of the inverse of the mean first passage time as a function of the damping constant $g_{0}^{2}$ ( see Eq.(9) ) in Fig.(1). The density of modes of the bath has been assumed to be of the standard Lorentzian form, $\mathcal{D}(\omega)=\frac{2}{\pi} \frac{1}{\tau_{c}}\left(\frac{1}{\omega^{2}+\tau_{c}^{-2}}\right)$ with vanishing internal correlation time $\tau_{c}$. Typically for the other scaled parameters we have used $k_{B} T=0.1$, the strength of the $\delta$-correlated external noise, $D=0.1$. The mean first passage times have been calculated by averaging over 10000 trajectories. We first show the continuous curve in Fig.(1) which represents the case of direct driving by the external $\delta$-correlated noise. What is immediately apparent is the loss

of turnover of the rate as one allows the variation of the dissipation constant $g_{0}^{2}$ from low to high friction regime. This is in sharp contrast to what is shown by the dotted curve in 
Fig.(1) which depicts the situation of bath modulation with the response function $\varphi\left(t-t^{\prime}\right)$ determined by Eq.(10). Both the curves ( continuous and dotted ) representing the open system are compared to that for the standard Kramers' turnover, i.e., when the dynamics is calculated in absence of the external noise ( dashed curve ). As expected the rate in the driven system ( be it directly or through bath modulation ) is always higher that in the undriven one. Fig.(1) also shows that although in the low damping region the direct driving causes a much higher rate, it is, however, the bath modulation which becomes more effective in inducing activated barrier crossing in the high friction regime. It is thus interesting to note that the barrier crossing dynamics of the system in contact with an external noise modulated bath captures the essential turnover features of the Kramers' dynamics of the closed system. We therefore realize that although open, the nature of the response function of the reservoir as well as the thermodynamic consistency condition (8), make the open system feel like a closed system.

In Fig.(2) we show the variation of inverse of the mean first passage time as a function of the strength of the external noise $D$, keeping all other parameter same as before but for $g_{0}^{2}=2.0$. It is apparent that the barrier crossing is more facilitated by modulating the bath than driving the system directly for higher values of external noise strength.

\section{B. The rate : Theoretical results vs. numerical simulation}

So far we have considered the full potential $V(x)=\frac{1}{4} x^{4}-\frac{1}{2} x^{2}$ and vanishingly small correlation times for external and internal noises for numerical computation. We now turn to our basic theoretical result Eq.(43) which is a generalization of Kramers' rate for bath modulated dynamics for intermediate to strong damping regime. To examine its validity we calculate the rate (43) as a function of the damping constant $g_{0}^{2}\left(g_{0}\right.$ is related to both $\gamma(t)$ and $\varphi(t)$ in Eqs.(9) and (10) respectively ) for several values of external noise strength $D$. The scaled barrier height $E_{0}$ and $k_{B} T$ have been set to 0.25 and 0.1 , respectively. The

results are plotted in Fig.(3) ( continuous curve ) and compared to the rate, inverse of the 
mean first passage time ( dotted curve ) calculated numerically using full potential for the set of parameter values ( as given in the earlier subsection ) with Eq.(3). In Figs.(4) and (5) we further compare our theoretical results ( continuous curve) with numerical simulation ( dotted curve ) for the variation of rate as a function of external noise strength and its correlation time, respectively for several values of damping constant. It is apparent from Figs.(3-5) that the theoretical and numerical results are in good agreement.

\section{CONCLUSIONS}

Based on a system-reservoir microscopic model where the reservoir is modulated by an external, stationary and Gaussian noise with arbitrary decaying correlation function, we have numerically analyzed the model and generalized the Kramers' theory to calculate the steady state rate of escape from a metastable well. The main conclusions of this study are as follows;

(i) We have shown that since the reservoir is driven by the external noise and the dissipative properties of the system depend on the reservoir, a simple connection between the dissipation and the response function of the medium due to the external noise can be established. This connection is important for realizing an effective temperature of the reservoir characterizing the stationary state of the thermodynamically open system, as well as an effective transmission factor controlling the rate. Both of these quantities depend on the strength and correlation time of the external noise.

(ii) Many of the earlier treatments of the rate concern direct (phenomenological) driving of the system and did not emphasize the question of thermodynamic consistency in the context of open systems. The present theory being microscopic the fluctuation-dissipation-like relation (8) remains an inbuilt characteristic of the model itself as an essential thermodynamic consistency condition.

(iii) Based on numerical simulation of the full model potential we show that one can recover the turnover features of the Kramers' dynamics when the external noise modulates 
the reservoir rather than the system directly. This recovery, we believe, is an offshoot of the derived thermodynamic consistency condition (8).

(iv) Provided the long time limit of the moments for the stochastic processes pertaining to the external and internal noises characterized by arbitrary decaying correlation functions exist, the expression for generalized Kramers' rate of barrier crossing for the open system we derive here is fairly general. We have shown that it agrees reasonably well with numerical simulation using full potential for this model.

The creation of a typical nonequilibrium open situation by modulating a bath with the help of an external noise is not an uncommon phenomenon in applications and industrial processing. The external agency generating noise does work on the bath by stirring, pumping, agitating, etc., to which the system dissipates internally. In the present treatment we are concerned with a nonequilibrium steady state which signifies a constant throughput of energy in contrast to thermal equilibrium defined by an constant temperature. We believe that these considerations are likely to be important in other related issues in nonequilibrium open systems and may serve as a basis for studying processes occurring within irreversibly driven environments [6, 35] and for thermal ratchet problems [22].

\section{ACKNOWLEDGMENTS}

We thank E. Pollak for his kind interest in this work and for pointing out the Ref. (35) to us. SKB in indebted to Council of Scientific and Industrial Research (CSIR), Government of India for financial support.

\section{APPENDIX A: CALCULATION OF VARIANCES}

We consider a particular case as an example where the external noise $\epsilon(t)$ is $\delta$-correlated and the internal noise is an Ornstein-Uhlenbeck process, i.e.,

$$
\left\langle\epsilon(t) \epsilon\left(t^{\prime}\right)\right\rangle_{e}=2 D \delta\left(t-t^{\prime}\right)
$$


and

$$
\left\langle f(t) f\left(t^{\prime}\right)\right\rangle=\frac{g_{0}^{2} k_{B} T}{\tau_{c}} e^{-\left|t-t^{\prime}\right| / \tau_{c}}
$$

Consequently, from the fluctuation-dissipation relation we derive the dissipative kernel as,

$$
\gamma\left(t-t^{\prime}\right)=\frac{g_{0}^{2}}{\tau_{c}} e^{-\left|t-t^{\prime}\right| / \tau_{c}}
$$

It should be noted that for $\tau_{c} \rightarrow 0$, the above noise process become $\delta$-correlated.

The Laplace transform of $\gamma(t)$ as given above can be written as

$$
\tilde{\gamma}(s)=\frac{g_{0}^{2}}{s \tau_{c}+1}
$$

and subsequently, we have for $\tau_{c} \neq 0$,

$$
\tilde{h}(s)=\frac{s+a}{s^{3}+a s^{2}+b s+c_{0}}
$$

where

$$
a=\frac{1}{\tau_{c}}, \quad b=\omega_{0}^{2}+\frac{g_{0}^{2}}{\tau_{c}} \text { and } c_{0}=\frac{\omega_{0}^{2}}{\tau_{c}} .
$$

We find that the inverse Laplace transform of $\tilde{h}(s)$ reads

$$
h(t)=c_{1} e^{-\Delta_{1} t}+c_{2} e^{-\Delta_{2} t} \sin (\beta t+\alpha)
$$

where the coefficients $c_{1}, c_{2}, \Delta_{1}, \Delta_{2}, \beta$ and $\alpha$ are given by

$$
\begin{aligned}
\Delta_{1} & =-\mathcal{A}-\mathcal{B}+\frac{a}{3} \\
\Delta_{2} & =\frac{1}{2}(\mathcal{A}+\mathcal{B})+\frac{a}{3} \\
\beta & =\frac{\sqrt{3}}{2}(\mathcal{A}-\mathcal{B}) \\
c_{1} & =\frac{1}{2 \Delta_{2}-\Delta_{1}-d} \\
d & =\frac{a\left(2 \Delta_{2}-\Delta_{1}\right)-\Delta_{2}^{2}-\beta^{2}}{a-\Delta_{1}} \\
\mathcal{A} & =\left(-\frac{a^{3}}{27}+\frac{a b}{6}-\frac{c_{0}}{2}+\sqrt{\mathcal{Q}}\right)^{1 / 3}
\end{aligned}
$$




$$
\begin{aligned}
\mathcal{B} & =\left(-\frac{a^{3}}{27}+\frac{a b}{6}-\frac{c_{0}}{2}-\sqrt{\mathcal{Q}}\right)^{1 / 3}, \\
c_{2} & =-\frac{c_{1}}{\beta}\left[\left(d-\Delta_{2}\right)^{2}+\beta^{2}\right]^{1 / 2}, \\
\alpha & =\tan ^{-1}\left(\frac{\beta}{d-\Delta_{2}}\right) \text { and } \\
\mathcal{Q} & =-\frac{a^{2} b^{2}}{108}+\frac{b^{3}}{27}+\frac{a^{3} c_{0}}{27}-\frac{a b c_{0}}{6}+\frac{c_{0}^{2}}{4} .
\end{aligned}
$$

Here we note that for a physically allowed solution $\Delta_{1}, \Delta_{2}$ must be positive. Since by Eq.(16) $h(t)$ depends on the memory kernel $\gamma(t)$ which is of decaying type and all the moments, in general, reach asymptotic constancy as shown in Sec. III, these quantities are positive (which depends on the correlation time $\tau_{c}$, the strength of the noise and other potential parameters) which may be checked (after some algebra) by considering the limiting cases such as $\tau_{c} \rightarrow 0$ and $\tau_{c} \rightarrow$ large.

Substituting Eq. (A1) into the expressions for variances [external noise is $\delta$-correlated], namely into (21) and (22) we have after some lengthy algebra

$$
\sigma_{x x}^{2}(t)=\sigma_{x x}^{2(i)}(t)+\sigma_{x x}^{2(e)}(t)
$$

where

$$
\begin{aligned}
\sigma_{x x}^{2(i)}(t)= & k_{B} T\left(c_{2} R+\frac{c_{1}}{\Delta_{1}}\right)\left[2-\omega_{0}^{2}\left(c_{2} R+\frac{c_{1}}{\Delta_{1}}\right)\right] \\
& +k_{B} T\left\{-\frac{c_{1}}{\Delta_{1}} e^{-\Delta_{1} t}\left[2-2 \omega_{0}^{2} c_{2} R-\frac{2 \omega_{0}^{2} c_{1}}{\Delta_{1}}+e^{-\Delta_{1} t}\left(\Delta_{1} c_{1}+\frac{\omega_{0}^{2} c_{1}}{\Delta_{1}}\right)\right]\right. \\
& -\frac{2 c_{2} e^{-\Delta_{2} t}}{\Delta_{2}^{2}+\beta^{2}}\left[1-\omega_{0}^{2} c_{2} R+\frac{\omega_{0}^{2} c_{1}}{\Delta_{1}}\left(e^{-\Delta_{1} t}-1\right)\right]\left[\Delta_{2} \sin (\beta t+\alpha)+\beta \cos (\beta t+\alpha)\right] \\
& -2 c_{1} c_{2} e^{-\left(\Delta_{1}+\Delta_{2}\right) t} \sin (\beta t+\alpha) \\
& -\frac{\Delta_{2} \beta \omega_{0}^{2} c_{2}^{2} e^{-2 \Delta_{2} t}}{\left(\Delta_{2}^{2}+\beta^{2}\right)^{2}} \sin 2(\beta t+\alpha)-\frac{\beta^{2} \omega_{0}^{2} c_{2}^{2} e^{-2 \Delta_{2} t}}{\left(\Delta_{2}^{2}+\beta^{2}\right)^{2}} \\
& \left.+\left[\frac{\omega_{0}^{2}\left(2 \beta^{2}-\Delta_{2}^{2}\right)}{\left(\Delta_{2}^{2}+\beta^{2}\right)^{2}}-1\right] c_{2}^{2} e^{-2 \Delta_{2} t} \sin ^{2}(\beta t+\alpha)\right\}
\end{aligned}
$$

with

$$
R=\frac{1}{\Delta_{2}^{2}+\beta^{2}}\left(\Delta_{2} \sin \alpha+\beta \cos \alpha\right)
$$


and

$$
\begin{aligned}
\sigma_{x x}^{2(e)}(t)= & 2 D\left(\frac{\kappa_{0}}{g_{0}} \tau_{c}\right)^{2}\left[c_{1}^{2}\left(\omega_{0}^{4}+\Delta_{1}^{4}+2 \omega_{0}^{2} \Delta_{1}^{2}\right) I_{A}(t)\right. \\
& +c_{2}^{2}\left\{\omega_{0}^{4}+\left(\Delta_{2}^{2}-\beta^{2}\right)^{2}-4 \beta^{2} \Delta_{2}^{2}+2 \omega_{0}^{2}\left(\Delta_{2}^{2}-\beta^{2}\right)\right\} I_{B}(t) \\
& +2 c_{1} c_{2}\left\{\omega_{0}^{4}+\Delta_{1}^{2}\left(\Delta_{2}^{2}-\beta^{2}\right)+\omega_{0}^{2}\left(\Delta_{1}^{2}+\Delta_{2}^{2}-\beta^{2}\right)\right\} I_{C}(t) \\
& \left.-2 c_{2}^{2} \beta \Delta_{2}\left(\Delta_{2}^{2}-\beta^{2}+\omega_{0}^{2}\right) I_{D}(t)+4 c_{2}^{2} \beta^{2} \Delta_{2}^{2} I_{E}(t)-4 c_{1} c_{2} \beta \Delta_{2}\left(\Delta_{1}^{2}+\omega_{0}^{2}\right) I_{F}(t)\right] .
\end{aligned}
$$

Here the I's are defined by

$$
\begin{aligned}
& I_{A}(t)=\int_{0}^{t} e^{-2 \Delta_{1} t} d t \\
& I_{B}(t)=\int_{0}^{t} e^{-2 \Delta_{2} t} \sin ^{2}(\beta t+\alpha) d t \\
& I_{C}(t)=\int_{0}^{t} e^{-\left(\Delta_{1}+\Delta_{2}\right) t} \sin (\beta t+\alpha) d t \\
& I_{D}(t)=\int_{0}^{t} e^{-2 \Delta_{2} t} \sin 2(\beta t+\alpha) d t, \\
& I_{E}(t)=\int_{0}^{t} e^{-2 \Delta_{2} t} d t \text { and } \\
& I_{F}(t)=\int_{0}^{t} e^{-\left(\Delta_{1}+\Delta_{2}\right) t} \cos (\beta t+\alpha) d t .
\end{aligned}
$$

Similarly

$$
\sigma_{v v}^{2}(t)=\sigma_{v v}^{2(i)}(t)+\sigma_{v v}^{2(e)}(t)
$$

where

$$
\begin{aligned}
\sigma_{v v}^{2(i)}(t)= & k_{B} T-\left[\left(\Delta_{1}^{2}+\omega_{0}^{2}\right) c_{1}^{2} e^{-2 \Delta_{1} t}+\beta^{2} c_{2}^{2} e^{-2 \Delta_{2} t}\right. \\
& -\beta \Delta_{2} c_{2}^{2} e^{-2 \Delta_{2} t} \sin 2(\beta t+\alpha)+\left(\Delta_{2}^{2}+\omega_{0}^{2}-\beta^{2}\right) c_{2}^{2} e^{-2 \Delta_{2} t} \sin ^{2}(\beta t+\alpha) \\
& \left.+e^{-\left(\Delta_{1}+\Delta_{2}\right) t}\left\{2 c_{1} c_{2}\left(\omega_{0}^{2}+\Delta_{1} \Delta_{2}\right) \sin (\beta t+\alpha)-2 \Delta_{1} \beta c_{1} c_{2} \cos (\beta t+\alpha)\right\}\right]
\end{aligned}
$$

and

$$
\begin{aligned}
\sigma_{v v}^{2(e)}(t)= & 2 D\left(\frac{\kappa_{0}}{g_{0}} \tau_{c}\right)^{2}\left[c_{1}^{2} \Delta_{1}^{2}\left(\omega_{0}^{2}+\Delta_{1}^{2}\right)^{2} I_{A}(t)\right. \\
& +c_{2}^{2}\left\{\left(\omega_{0}^{2}+\Delta_{2}^{2}-3 \beta^{2}\right)^{2} \Delta_{2}^{2}-\left(\omega_{0}^{2}+3 \Delta_{2}^{2}-\beta^{2}\right)^{2} \beta^{2}\right\} I_{B}(t) \\
& +2 c_{1} c_{2} \Delta_{1} \Delta_{2}\left(\omega_{0}^{2}+\Delta_{1}^{2}\right)\left(\omega_{0}^{2}-3 \beta^{2}+\Delta_{2}^{2}\right) I_{C}(t)
\end{aligned}
$$




$$
\begin{aligned}
& -c_{2}^{2} \beta \Delta_{2}\left(3 \Delta_{2}^{2}-\beta^{2}+\omega_{0}^{2}\right)\left(\omega_{0}^{2}-3 \beta^{2}+\Delta_{2}^{2}\right) I_{D}(t) \\
& +c_{2}^{2} \beta^{2}\left(\omega_{0}^{2}-\beta^{2}+3 \Delta_{2}^{2}\right) I_{E}(t) \\
& \left.-2 c_{1} c_{2} \beta \Delta_{1}\left(\Delta_{1}^{2}+\omega_{0}^{2}\right)\left(\omega_{0}^{2}+3 \Delta_{2}^{2}-\beta^{2}\right) I_{F}(t)\right]
\end{aligned}
$$

where, I's are defined in Eq.(A6a-A6t). The explicit expression for $\sigma_{x v}^{2}(t)$ can be derived from Eq.(23). In the limit $t \rightarrow \infty$ we calculate the stationary values of the variances. The variances $\sigma_{x x}^{2}(\infty), \sigma_{v v}^{2}(\infty)$ and $\sigma_{x v}^{2}(\infty)$ yield $\phi(\infty)$ and $\psi(\infty)$ and other relevant quantities. 


\section{REFERENCES}

[1] H. A. Kramers, Physica (Amsterdam) 7, 284 (1940).

[2] N. G. van Kampen, Prog. Theo. Phys. 64, 389 (1978).

[3] R. F. Grote and J. T. Hynes, J. Chem. Phys. 73, 2715 (1980).

[4] P. Hänggi and F. Mojtabai, Phys. Rev. A 26, 1168 (1982).

[5] E. Pollak, J. Chem. Phys. 85, 865 (1986).

[6] J. Ray Chaudhuri, G. Gangopadhyay and D. S. Ray, J. Chem. Phys. 109, 5565 (1998).

[7] S. K. Banik, J. Ray Chaudhuri and D. S. Ray, J. Chem. Phys. 112, 8330 (2000).

[8] A. O. Caldeira and A. J. Leggett, Phys. Rev. Lett. 46, 211 (1981).

[9] H. Grabert, P. Schramm and G. L. Ingold, Phys. Rep. 168, 115 (1988).

[10] H. Grabert, U. Weiss and P. Hänggi, Phys. Rev. Lett. 52, 2193 (1984).

[11] J. Ray Chaudhuri, B. C. Bag and D. S. Ray, J. Chem. Phys. 111, 10852 (1999).

[12] P. Hänggi, P. Talkner and M. Borkovec, Rev. Mod. Phys. 62, 251 (1990).

[13] V. I. Mel'nikov, Phys. Rep. 209, 1 (1991).

[14] P. Talkner, E. Pollak and A. M. Berezhkovskii, Eds., Chem. Phys. 235, 1 (1998).

[15] U. Weiss, Quantum Dissipative Systems (World Scientific, Singapore, 1993).

[16] G. W. Ford, M. Kac and P. Mazur, J. Math. Phys. 6, 504 (1965).

[17] W. H. Louisell, Quantum Statistical Properties of Radiation (Wiley, New York, 1973).

[18] R. Zwanzig, J. Stat. Phys. 9, 215 (1973).

[19] K. Lindenberg and V. Seshadri, Physica A 109, 483 (1981).

[20] R. Kubo, M. Toda and N. Hashitsume, Statistical Physics II, Nonequilibrium Statistical 
Mechanics (Springer, Berlin, 1985).

[21] K. Lindenberg and B. J. West, The Nonequilibrium Statistical Mechanics of Open and Closed Systems (VCH Publisher, Inc., New York, 1990).

[22] R. D. Astumian, Science 276, 917 (1997) and the references given therein.

[23] K. M. Rattray and A. J. McKane, J. Phys. A 24, 4375 (1991).

[24] See, for example, F. Moss and P. V. E. McClintock, Eds., Noise in nonlinear dynamical systems, Vol. I-III (Cambridge University Press, England, 1989).

[25] J. Masoliver and J. M. Porrà, Phys. Rev. E 48, 4309 (1993).

[26] W. Horsthemke, C. R. Doering, T. S. Ray and M. A. Burschka, Phys. Rev. A 45, 5492 (1992).

[27] S. J. B. Einchcomb and A. J. McKane, Phys. Rev. E 49, 259 (1994).

[28] W. Horsthemke and R. Lefever, Noise-Induced Transitions (Springer-Verlag, Berlin, 1984).

[29] L. Farkas, Z. Phys. Chem. (Leipzig) 125, 236 (1927).

[30] J. M. Bravo, R. M. Velasco and J. M. Sancho, J. Math. Phys. 30, 2023 (1989).

[31] S. A. Adelman, J. Chem. Phys. 64, 124 (1976).

[32] R. M. Mazo in Stochastic Processes in Nonequilibrium Systems, edited by L. Garrido, P. Segler and P. J. Shepherd, Lecture Notes in Physics, Vol. 84 (Springer-Verlag, Berlin, 1978).

[33] B. Carmeli and A. Nitzan, J. Chem. Phys. 29, 1481 (1984).

[34] R. F. Fox, Phys. Rev. A 43, 2649 (1991).

[35] R. Hernandez, J. Chem. Phys. 111, 7701 (1999). 


\section{FIGURES}

FIG. 1. Plot of barrier crossing rate, $k$ vs. damping constant, $g_{0}^{2}$. The solid and the dotted line correspond to direct driving of the system and bath modulation, respectively $(D=0.1)$. The dashed line corresponds to the thermodynamically closed system, i.e., the system without any external driving $(D=0.0) . k_{B} T=0.1$ is common for all the three curves. (units are arbitrary)

FIG. 2. Plot of barrier crossing rate, $k$ vs. external noise strenth, D for a constant $g_{0}^{2}$. The curve (a) represents the results for bath modulation while the curve (b) is the result for direct additive driving. (units are arbitrary)

FIG. 3. Plot of barrier crossing rate, $k$ vs. damping constant, $g_{0}^{2}$ for different external noise strengths D. The solid lines correspond to theoretical result (Eq.(52)) and the dotted curves are due to simulation. (a) $D=0.15$, (b) $D=0.10$ and (c) $D=0.05$. (units are arbitrary)

FIG. 4. Plot of barrier crossing rate, $k$ vs. external noise strength, $D$ for different values of

$g_{0}^{2}$. The solid and the dotted lines are same as in Fig.(3). (a) $g_{0}^{2}=2.0$ and (b) $g_{0}^{2}=3.0$. (units are arbitrary)

FIG. 5. Plot of barrier crossing rate, $k$ vs. correlation time of the external noise, $\tau_{e}$ for different values of external noise strengths, $D$. The solid and the dotted lines are same as in Fig.(3). (a) $D$ $=0.5$, (b) $D=1.0$ and (c) $D=1.5$. (units are arbitrary) 


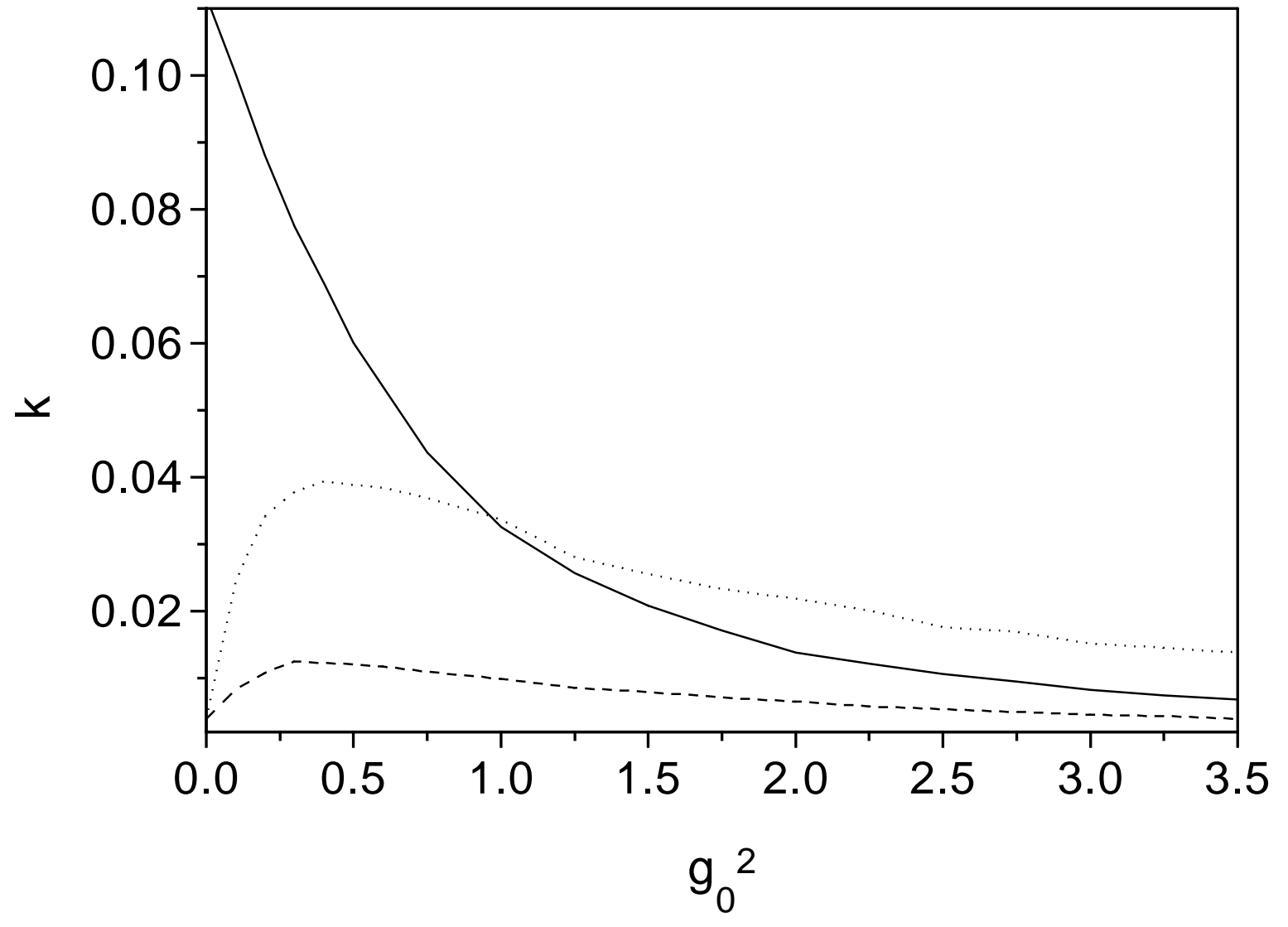

Fig.(1) 


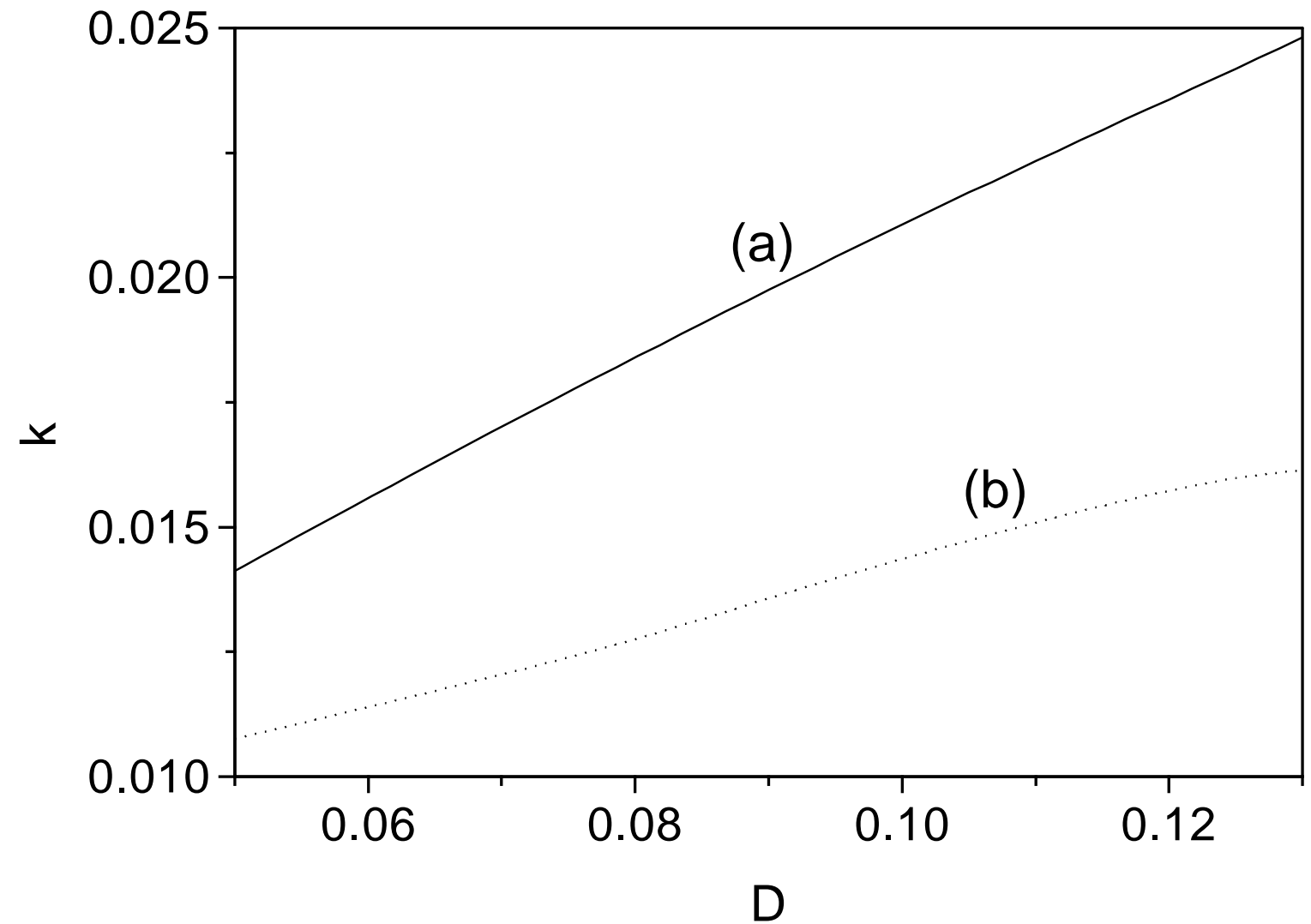

Fig.(2) 


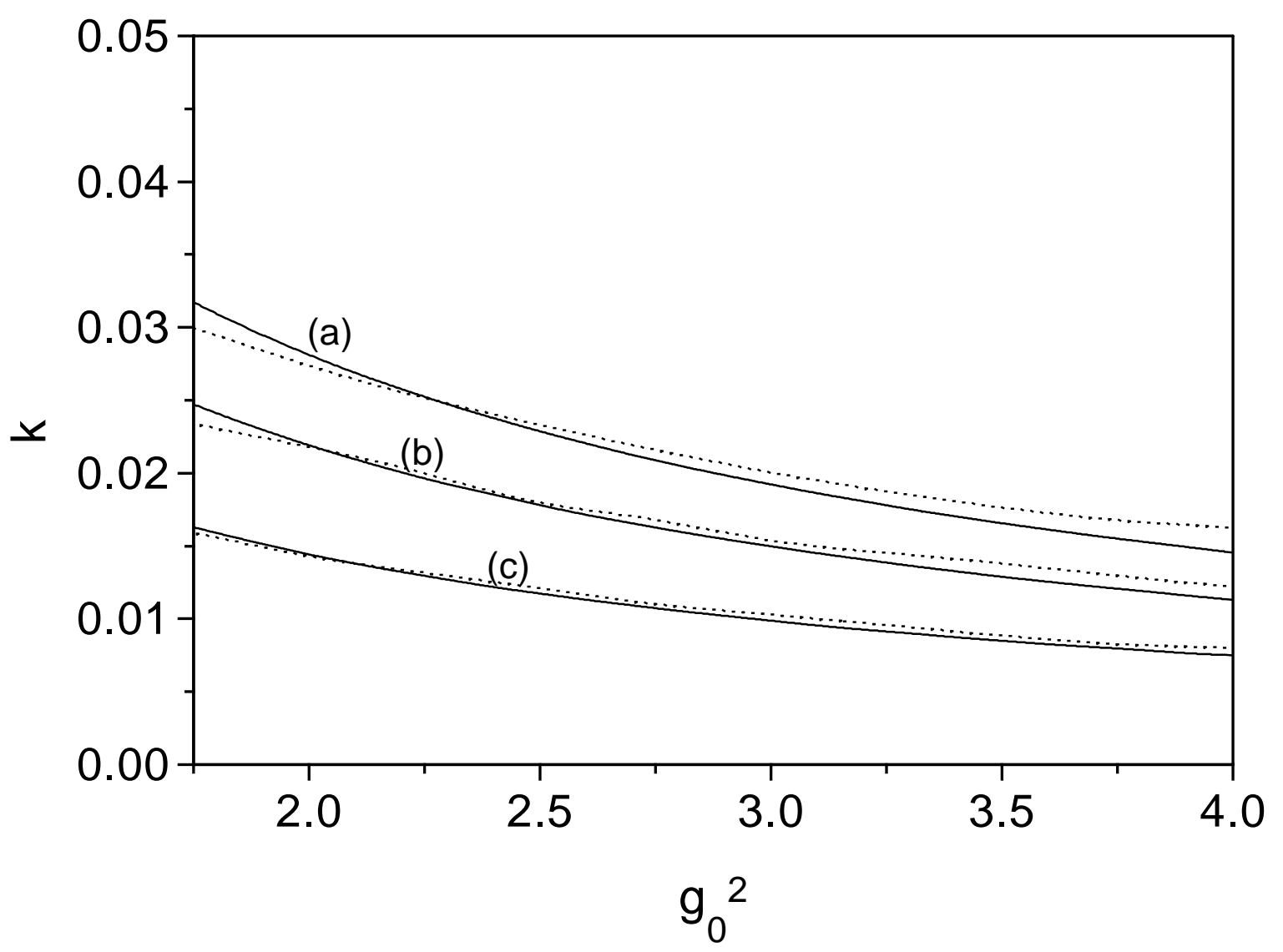

Fig.(3) 


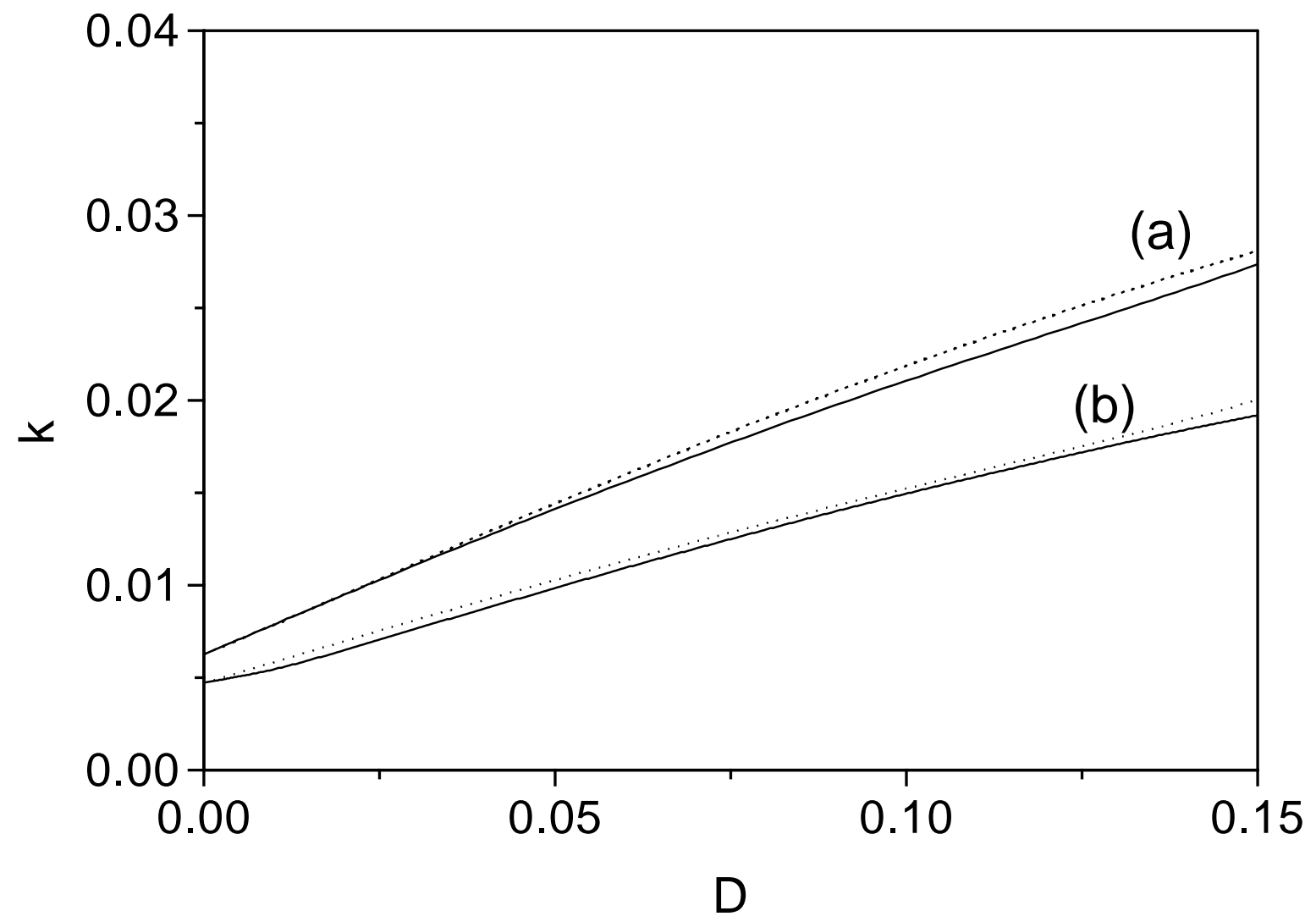

Fig.(4) 


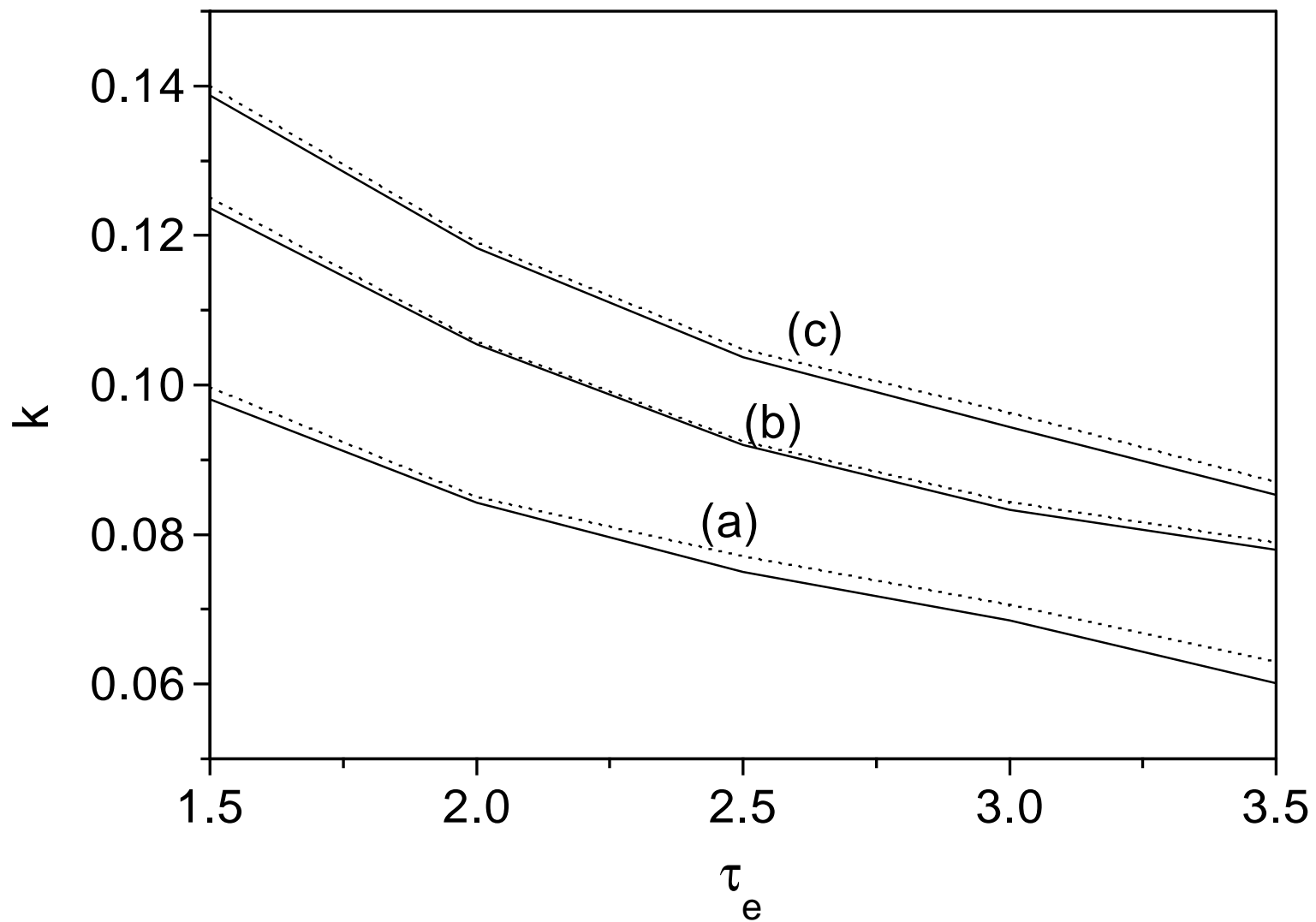

Fig.(5) 